\title{
Redundancy concepts for brake and CV steering in highly automated commercial vehicles
}

Dr. Falk Hecker, Knorr-Bremse Systeme für Nutzfahrzeuge GmbH

This manuscript was not available on completion of this publication.

Thank you for your understanding. 